\title{
The effects of electrical stimulation or an electrolytic lesion in the mediodorsal thalamus of the rat on survival, body weight, food intake and running activity in the activity-based anorexia model
}

\author{
Laura Luyten $^{1 \dagger}$, Marleen Welkenhuysen ${ }^{1 \dagger}$, Kris van Kuyck ${ }^{1 \dagger}$, Steffen Fieuws ${ }^{2}$, John Das ${ }^{1}$, Raf \\ Sciot $^{3}$, Bart Nuttin ${ }^{1 \S}$ \\ ${ }^{\dagger}$ These authors contributed equally to the study. ${ }^{1}$ Laboratory of Experimental Functional Neurosurgery, \\ Department of Neurosciences, K.U.Leuven, Provisorium I, Minderbroedersstraat 19 bus 1033, 3000 Leuven, \\ Belgium. ${ }^{2}$ Biostatistical Centre, U.Z. Sint-Rafaël, Kapucijnenvoer 35, 3000 Leuven, Belgium. ${ }^{3}$ Morphology and \\ Molecular Pathology Section, Minderbroedersstraat 12, 3000 Leuven, Belgium. ${ }^{\S}$ Corresponding author: Bart \\ Nuttin - Contact address: Neurosurgery, U.Z. Leuven, Herestraat 49 - bus 7003, 3000 Leuven, Belgium Tel.: \\ +33 16 344290; fax: +32 16344285
}

Published in Brain Research Bulletin, 2009, 79(2): p. 116-122

\section{ABSTRACT}

The glucose metabolism in the mediodorsal thalamus (MD) is increased in rats in the activity-based anorexia (ABA) model. In patients, electrical stimulation in hyperactive brain regions reduced symptoms in e.g. major depressive disorder and cluster headache. In two blinded randomised controlled experiments, we therefore examined the effects of high-frequency electrical stimulation and an electrolytic lesion in the MD in a validated rat model for anorexia nervosa. The ABA model was successfully replicated in all our experiments, with a reduction in body weight, food intake, and survival time and an increase in running activity.

In a first experiment, we evaluated the effect of electrical stimulation or a curative lesion in the MD on survival, body weight, food intake and locomotor activity in ABA rats. Electrical MD stimulation or an electrolytic MD lesion did not improve the symptoms of rats in the ABA model, compared to control groups.

In a second experiment, we investigated the effect of a preventive electrolytic lesion in the MD on rats in the ABA model. Although there was no significant improvement of survival, body weight and food intake, locomotor activity was significantly reduced in the lesion group compared to the control group. Apart from this positive effect on running activity, we found no convincing evidence for the suitability of the MD as a neuromodulation target for anorexia nervosa patients.

\section{INTRODUCTION}

Anorexia nervosa (AN) is a severe psychiatric disorder characterized by a refusal to maintain body weight at or above a minimally normal weight for age and height, an intense fear of gaining weight, and a disturbance in the way one's body weight and shape are experienced. In postmenarcheal females, there is an absence of at least three consecutive menstrual cycles [1]. In addition, the lifetime occurrence of excessive exercise is between 75 and $84 \%$ [6]. 
Despite intensive treatment programs combining nutritional therapy with psychotherapy and/or pharmacotherapy, around $20 \%$ of the patients continues to suffer chronically. In accordance, mortality rates are high [41], with physical complications accompanying extreme starvation and suicide as the most common reported causes of death. Therefore, developing new treatment options is of utmost importance [31].

The ABA model is one of the best characterized animal models for AN and has been used for investigating potential new AN treatments (e.g. [9]). In this model, food restriction to $1.5 \mathrm{~h}$ daily in the presence of a running wheel leads to the development of hyperactivity and a spontaneous restriction of food intake, leading to severe emaciation and often death [29]. This behaviour models some of the core symptoms in AN patients, e.g. dieting, loss of body weight and excessive exercising [12].

On the other hand, AN is characterized by many psychological symptoms. Although many hold expectations that all features of AN including psychological, physiological, and psychosocial symptoms should be modelled, an animal model will never provide enough psychological complexity to explain truly the associated human psychopathology [28]. However, this observation does not alter the fact that an animal model can be very useful to study certain aspects of the human disease. Moreover, the ABA model has been used extensively and it has been argued to be the best animal model available of AN [8].

Functional neuroimaging and neuroendocrinological studies provide substantial evidence for the involvement of the central nervous system in the mediation and maintenance of the symptoms in AN [30]. We therefore speculate that therapeutic interventions directed at the central nervous system, like electrical brain stimulation, may alleviate symptoms in patients with treatment-resistant AN. Likewise, electrical brain stimulation and lesioning have proven to be successful in patients suffering from treatment-resistant obsessive-compulsive disorder and major depressive disorder, two psychiatric disorders related to $\mathrm{AN}[21,24,26]$. However, in $\mathrm{AN}$ it is unknown in which brain targets neuromodulation may relieve symptoms.

Previous results from a functional neuroimaging study [36] in which the cerebral ${ }^{18} \mathrm{~F}$ fluorodeoxyglucose (FDG) uptake of rats in the ABA model was compared to normal rats, revealed certain hyperactive regions, among which the mediodorsal thalamus (MD). Based upon these findings and since this target selection strategy has been successful in major depressive disorder [21], we chose the MD as a neuromodulation target in the present study.

We hypothesize that an electrolytic lesion and/or electrical brain stimulation in the mediodorsal thalamus increases survival, body weight and food intake, and reduces locomotor activity. 


\section{MATERIALS AND METHODS}

\subsection{Experiment 1}

\subsubsection{Subjects and housing}

Experiments were conducted on 48 female Wistar rats weighing 200-250 g at arrival. They were housed individually with food and water ad libitum available. A 12:12 h light/dark cycle was imposed (lights on at 7:00 a.m.). The temperature in the testing room was kept constant at $\pm 20^{\circ} \mathrm{C}$. All experiments were carried out in accordance with protocols approved by the local university animal ethics committee and in accordance with the European Communities Council Directive of November 24, 1986 (86/609/EEC).

\subsubsection{Experimental groups}

Rats were divided into 5 groups. The first group (stim $M D+A B A$ ) was stimulated in the MD, the second one (no stim $M D+A B A$; negative control) was not stimulated, the third one (stim $M D-A B A$; ABA-specificity control) was stimulated in the MD, but had $24 \mathrm{~h}$ access to food, the fourth one (stim random $+A B A$; MD-specificity control) was stimulated in a random nucleus and the fifth group (lesion $M D+A B A$ ) received a lesion in the $M D$.

\subsubsection{Surgery}

Monopolar platinum-iridium stimulation electrodes (200 $\mu \mathrm{m}$ diameter) with a bare transversally cut tip were stereotactically implanted under general anaesthesia $(0.5 \mathrm{~g} / \mathrm{kg}$ chloral hydrate, s.c.; Sigma C8383, Steinheim, Germany) bilaterally in the MD (stim $M D+A B A$, no stim $M D+A B A$ and stim $M D-$ $A B A$ groups; $2.9 \mathrm{~mm}$ posterior to bregma, $2.2 \mathrm{~mm}$ lateral to the midline, $5.3 \mathrm{~mm}$ ventral to the dural surface, $15^{\circ}$ angle to the sagittal plane [27]) or in a region elsewhere in the brain not activated on PET [36] (stim random $+A B A$ group) (caudate putamen, cingulate area 2, septofimbrial nucleus, CA1 hippocampus, peduncular part of lateral hypothalamus, external globus pallidus, zona incerta and substantia nigra reticular part, one rat for each region). For the production of electrolytic lesions (lesion $M D+A B A$ group), insulated stainless steel electrodes ( $200 \mu \mathrm{m}$ diameter) with a bare tip of 0.5 $\mathrm{mm}$ were implanted bilaterally in the MD (coordinates, see above). In order to keep the observer blind for group division during subsequent testing, two connectors were mounted on the skull of all animals. Those connectors conducted the current in case of electrical stimulation or lesioning. The surgery and introduction in the ABA cage were on diestrus 2 of the oestrus cycle as determined by light microscopy evaluation (x10 and x20 magnification; Leica DM LA; Leica Microsystems, Wetzlar, Germany) of vaginal smears [19]. The oestrus cycle may indeed affect food intake and motor activity [4]. 


\subsubsection{ABA model}

All experiments were conducted in custom-made ABA cages $(0.36 \times 0.36 \times 0.36 \mathrm{~m})$ with a running wheel (Ø $0.35 \mathrm{~m})$ (Campden Instruments, Loughborough, UK). An electro-magnetic rotary encoder (TWK-Elektronik GmbH, Düsseldorf, Germany) was mounted on the shaft of the running wheel for continuous registration of the position of the running wheel with a digital I/O card (National Instruments, Austin, Texas, USA) and LabVIEW 7.0 (National Instruments).

Eight days after surgery, rats were introduced in the ABA cages with $1.5 \mathrm{~h}$ access to $50 \mathrm{~g}$ of food daily starting at 9.30 a.m., except for the stim $M D-A B A$ group which had $24 \mathrm{~h}$ access to food. In all groups, daily food intake $(\mathrm{g})$ and body weight $(\mathrm{g})$ were measured at $11 \mathrm{a} . \mathrm{m}$. Water was ad libitum available during the whole day. The intervention started when body weight decreased to less than $85 \%$ compared to body weight at introduction in the ABA cage. From the intervention moment on, rats in the stim $M D+A B A$, stim random $+A B A$ and stim $M D-A B A$ groups were bilaterally stimulated with biphasic rectangular pulses of $60 \mu$ s per phase at $100 \mathrm{~Hz}$, with for each rat the highest amplitude that caused no visible disturbance of normal behaviour during a 3-minute observation period. Rats from the stim MD-ABA group did not develop activity-based anorexia, because they were not foodrestricted. These rats never reached the intervention criterion $(<85 \%$ body weight), so they were assigned a "sister" in the stim $M D+A B A$ group. For each rat of the stim $M D-A B A$ group, stimulation was started at the same moment as for its "sister". In rats of the lesion $M D+A B A$ group, bilateral electrolytic lesions were produced at the intervention moment, through the already implanted electrodes with the same lesioning parameters as in experiment 2 (see below). Rats that were not stimulated (lesion $M D+A B A$ group and no stim $+A B A$ group) were also connected with cables to the stimulator in order to keep the observer blind for group division during subsequent testing.

\subsubsection{Euthanasia and histology}

At the end of the experiment (i.e. when the body weight was decreased to $\leq 70 \%$ of the initial body weight or after 10 days of intervention), animals were euthanized with an overdose of Nembutal ( $3 \mathrm{ml}$, i.p.; CEVA Santé Animale, Brussels, Belgium) and brains were processed for paraffin coronal sectioning $(5 \mu \mathrm{m})$. Brain slices were stained with cresyl violet (Merck KGaA, Darmstadt, Germany) and examined under a light microscope. Lesion volumes were estimated for both lesions in each rat of the lesion $M D+A B A$ group (experiment 1) and the lesion group (experiment 2). The antero-posterior depth of the lesion was calculated as the distance between the first and the last histological slices which showed a section of the lesion. In addition, the maximal medio-lateral width and dorso-ventral height of the lesions on the histological slice with the largest lesion area were determined. The approximate lesion volume $\left(\mathrm{mm}^{3}\right)$ was calculated using the ellipsoid formula $(\mathrm{V}=\mathrm{DWH} \pi / 6$, where $\mathrm{V}$ is volume, $\mathrm{D}$ is depth, $\mathrm{W}$ is width, and $\mathrm{H}$ is height) [2]. For each group the mean and standard deviation of lesion volumes were calculated. Bilateral lesion volume was correlated with survival 
(Pearson correlation) to exclude an effect of lesion size on survival. Rats were included in statistical analyses when the tips of the electrodes or the lesions were bilaterally located in the target region.

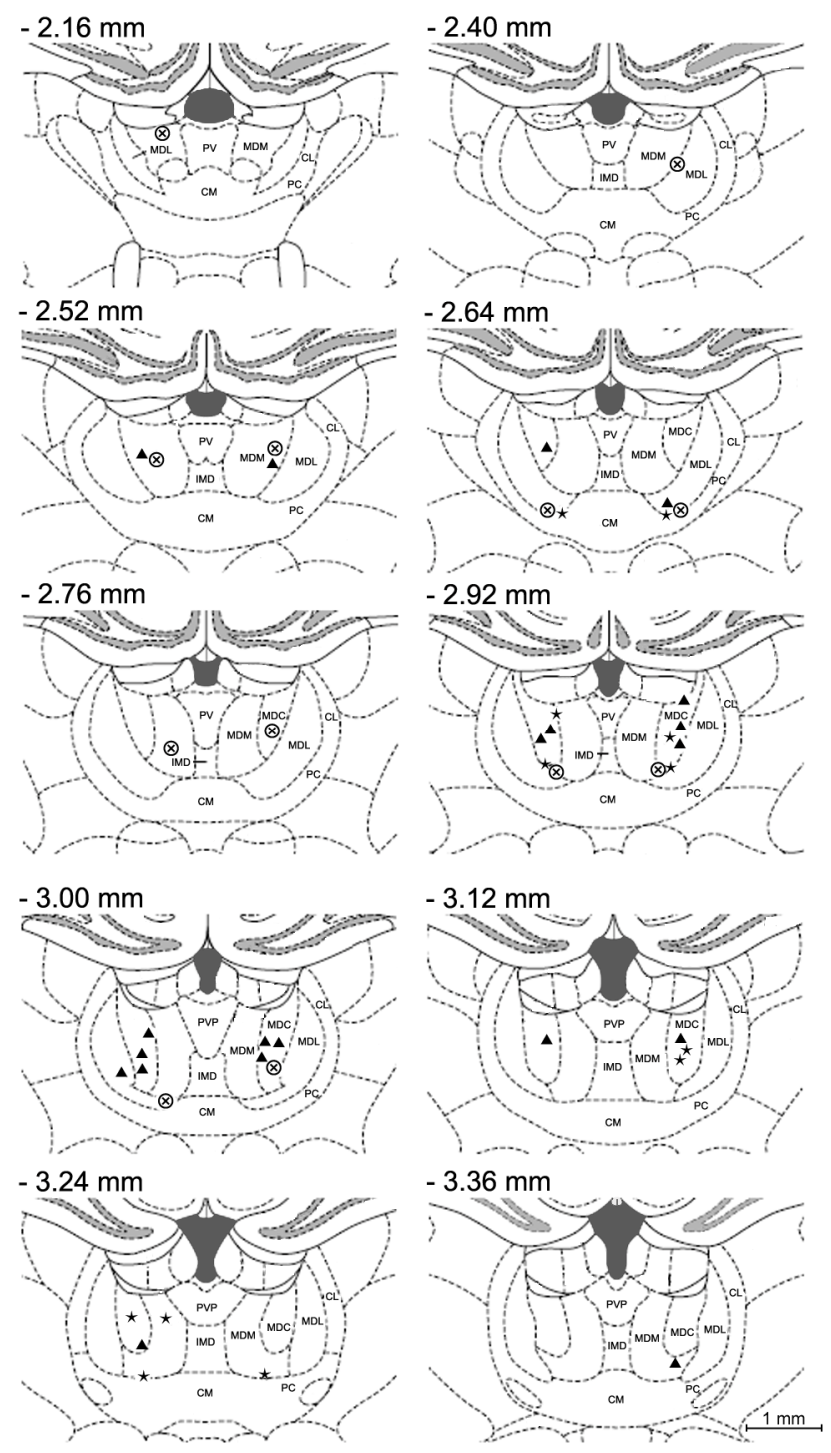

Fig. 1: Localization of the electrode tips in the stim $M D+A B A$ group $(\star)$, the stim $M D-A B A$ group $(\otimes)$, and the no stim $M D+A B A$ group $(\mathbf{\Delta})$. Distance of the coronal sections posterior to bregma is indicated. Adapted from [27]. CL: centrolateral thalamic nucleus; CM: central medial thalamic nucleus; IMD: intermediodorsal thalamic nucleus; MDC: mediodorsal thalamic nucleus, central part; MDL: mediodorsal thalamic nucleus, lateral part; MDM: mediodorsal thalamic nucleus, medial part; PC: paracentral thalamic nucleus; PV: paraventricular thalamic nucleus; PVP: paraventricular thalamic nucleus, posterior part.

\subsection{Experiment 2}

Experiment 2 was performed in the same way as experiment 1, with minor differences:

Experiments were conducted on 16 male Wistar rats weighing 300-350 g at arrival. Rats were divided into 2 groups: lesion and control.

MD lesions were made during surgery (lesion group; $0.8 \mathrm{~mm}$ posterior to bregma, $2.2 \mathrm{~mm}$ lateral to the midline, $4.8 \mathrm{~mm}$ ventral to the dural surface) with an anodal direct current pulse of $0.5 \mathrm{~mA}$ during 15 seconds. After lesioning, the electrodes were removed. In the control group, the skin was incised and burr holes were made but no electrodes were inserted. At the end of surgery, the wound was sutured.

The experiment ended when body weight was decreased to $\leq 70 \%$ of the initial body weight or when the rats developed a stable body weight (body weight on day $x+4>$ body weight on day $x$ [34]). Cryostat sections $(25 \mu \mathrm{m})$ were made instead of paraffin sections.

\subsection{Statistical analysis}

The average \% body weight (body weight on day $\mathrm{x} /$ body weight on the day of introduction in the ABA cage), $\%$ food intake (food intake on day $\mathrm{x} /$ body weight on day $\mathrm{x}$ ) and running activity (the number of wheel revolutions during the 24 hours preceding the measurements on day x) observed after start of the intervention were compared between groups using multivariate regression models, with group as a factor and a heterogeneous autoregressive covariance structure to account for the within-rat 
correlation over time. P-values for multiple comparisons between groups were adapted using Tukey's procedure. A comparison of average time trends was not considered. A natural logarithm (log) was used as transformation for the number of wheel revolutions to obtain a symmetric distribution of the residuals in the model. The analyses were performed using the SAS procedure PROC MIXED, which can cope with a varying number of observations between rats. Missingness at random was assumed. Details on this statistical approach can be found in Verbeke and Molenberghs [37]. Reported p-values are two-sided and were considered significant if $\mathrm{P}<0.05$.

The survival time (defined as time to the day that body weight decreased to $\leq 70 \%$ of initial body weight) was compared between groups using an exact log-rank test (using the package StatXact 3.0). Survival times were censored in experiment 1 if rats did not reach the $70 \%$ body weight criterion during the intervention period of maximally 10 days $(n=9)$ or in case of premature termination of the experiment $(n=3)$. Survival times were censored in experiment 2 if rats developed a stable body weight (body weight on day $\mathrm{x}+4>$ body weight on day $\mathrm{x}$ ) and thus considered to survive the experimental period $(n=10)$ or in case of premature termination of the experiment (n $=1$ ). Bonferroni correction was applied for multiple testing, P-values smaller than 0.005 are considered statistically significant.

\section{RESULTS}

\subsection{Experiment 1}

The average stimulation amplitudes (the highest amplitudes that caused no visible disturbance of normal behaviour during a 3minute observation period) are $1272 \mu \mathrm{A}$ (range: $470 \mu \mathrm{A}-2700 \mu \mathrm{A}$ ) in the stim
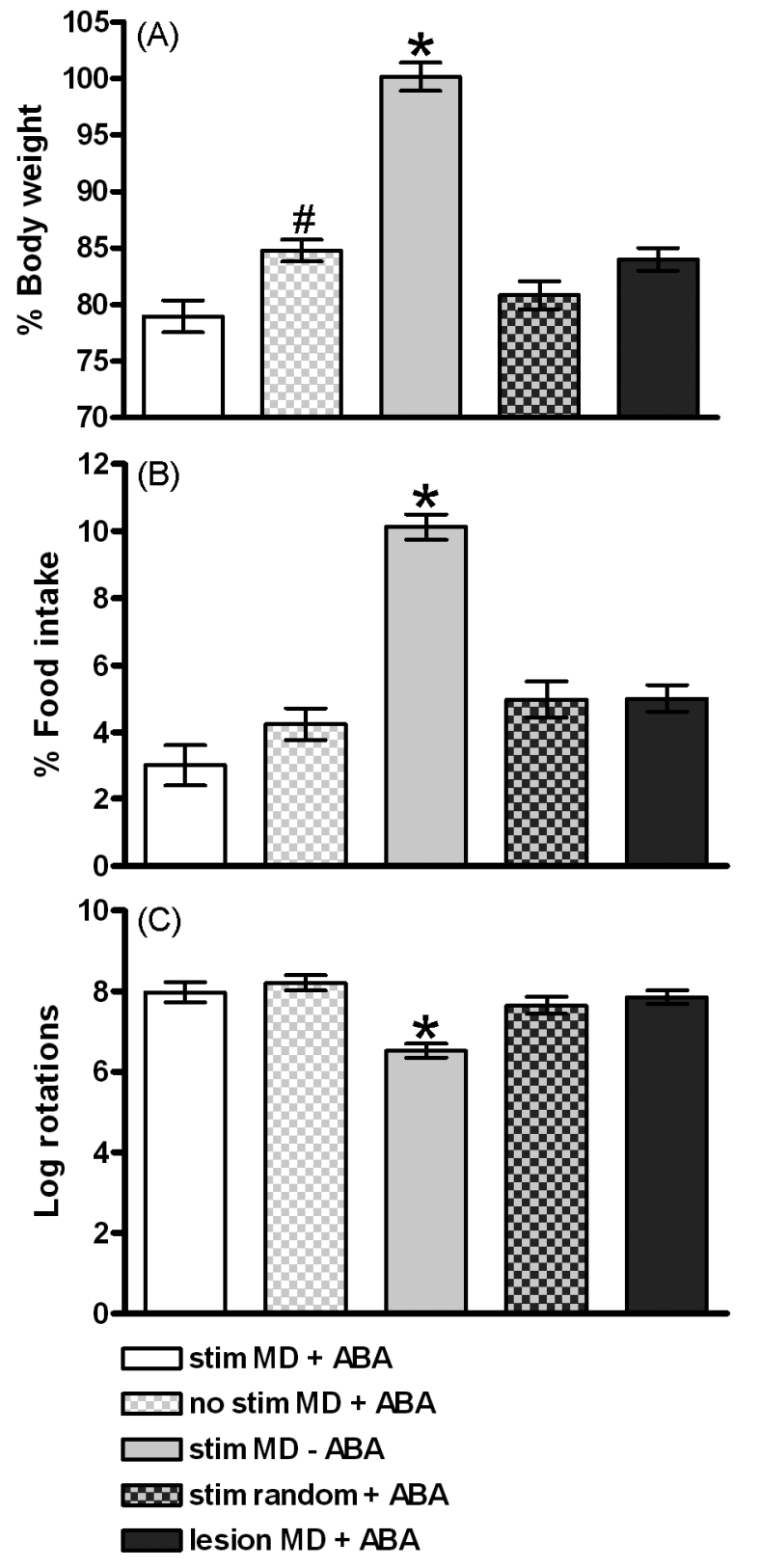

Fig. 2: Estimates of the mean ( \pm standard error) percentage body weight (A), percentage food intake (B) and log rotations $(\mathrm{C})$ in all groups.

*Significantly different from all other groups at $\mathrm{P}<0.01$. \#Significantly different from the stim $M D+A B A$ group at $\mathrm{P}$ $=0.0165$.

Stim: electrical stimulation; +ABA: $24 \mathrm{~h}$ running wheel and $1.5 \mathrm{~h}$ food availability daily; -ABA: $24 \mathrm{~h}$ running wheel and $24 \mathrm{~h}$ food availability daily. 
$M D+A B A$ group, $1650 \mu \mathrm{A}$ (range: $700 \mu \mathrm{A}-2500 \mu \mathrm{A}$ ) in the stim $M D-A B A$ group, and $1158 \mu \mathrm{A}$ (range: $200 \mu \mathrm{A}-2400 \mu \mathrm{A}$ ) in the stim random $+A B A$ group. These amplitudes are not significantly different between the 3 groups (Kruskal-Wallis, $\mathrm{P}=0.35$ ). Visible disturbances of normal behaviour induced by stimulation with maximal stimulation amplitudes during the 3-min observation period included extreme salivation, facial and oral activity, head bobbing, chasing its own tail, forelimb clonus, narrowing the eyes and rearing accompanied by spastic head movements. The localization of the electrode tips targeted to the MD is shown on Fig. 1.

In the lesion $M D+A B A$ group, the unilateral lesion volume (mean \pm standard deviation) was $1.01 \mathrm{~mm}^{3}$ $\pm 1.19 \mathrm{~mm}^{3}$. There was no significant correlation of bilateral lesion extent with survival $(\mathrm{r}=0.45, \mathrm{P}>$ $.05)$.

In the ' $+A B A$ ' groups, body weight decreased to less than $85 \%$ after $7 \pm 2.3$ days (mean \pm standard deviation) in the ABA cage. The stim $M D-A B A$ rats never reached this weight loss criterion, since they were not food-restricted.

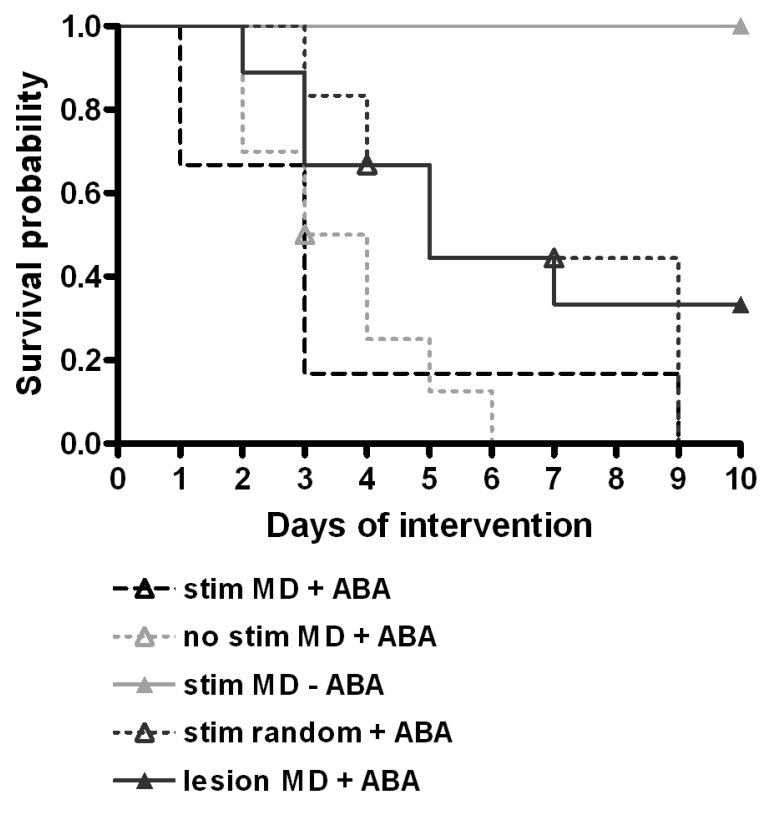

Fig. 3: Kaplan-Meier plot illustrating survival probability at daily intervals over a 10-day intervention period. At the start of intervention, $\mathrm{n}=6, \mathrm{n}=10, \mathrm{n}=6, \mathrm{n}=6$ and $\mathrm{n}=9$ for the stim $M D+A B A$ group, the no stim $M D+A B A$ group, the stim $M D-A B A$ group, the stim random $+A B A$ group and the lesion $M D+A B A$ group, respectively. Censored observations are indicated with a triangle.

Stim: electrical stimulation; $+\mathrm{ABA}: 24 \mathrm{~h}$ running wheel and $1.5 \mathrm{~h}$ food availability daily; -ABA: $24 \mathrm{~h}$ running wheel and $24 \mathrm{~h}$ food availability daily.

Percentage body weight (Fig. 2A) is significantly higher $(\mathrm{P}<0.01)$ in the stim $M D-A B A$ group compared to all other groups and in the no stim $M D+A B A$ group compared to the stim $M D+A B A$ group $(\mathrm{P}=$ 0.0165). Percentage food intake (Fig. 2B) is significantly higher $(\mathrm{P}<0.01)$ in the stim $M D-A B A$ group compared to all other groups. Locomotor activity is (Fig. 2C) significantly lower $(\mathrm{P}<0.01)$ in the stim $M D-A B A$ group compared to all other groups. The distances run per day were 4.2 $\mathrm{km}( \pm 1.4 \mathrm{~km})$ in the stim $M D+A B A$ group, $4.7 \mathrm{~km}( \pm 1.6 \mathrm{~km})$ in the no stim $M D+A B A$ group, $0.9 \mathrm{~km}( \pm 0.2 \mathrm{~km})$ in the stim $M D_{-}$ $A B A$ group, $3.4 \mathrm{~km}( \pm 2.5 \mathrm{~km})$ in the stim random $+A B A$ group and $3.8 \mathrm{~km}( \pm 1.8 \mathrm{~km})$ in the lesion $M D+A B A$ group (distances are means ( \pm standard deviations) per group, calculated from raw data averaged per rat).

Log-rank analysis indicates no significantly

increased survival of the stim $M D+A B A$ group compared to the no stim $M D+A B A$ group, stim random $+A B A$ group or lesion $M D+A B A$ group (Fig. 3). On the other hand, the survival probability is 
significantly higher in the stim $M D-A B A$ group compared to the stim $M D+A B A$ group $(\mathrm{P}=0.0022)$ and the no stim $M D+A B A$ group $(\mathrm{P}=0.0001)$.

\subsection{Experiment 2}

In the lesion group, the unilateral lesion volume (mean \pm standard deviation) was $0.93 \mathrm{~mm}^{3} \pm 0.82$ $\mathrm{mm}^{3}$. There was no significant correlation of bilateral lesion extent with survival $(\mathrm{r}=-0.08, \mathrm{P}>.05)$.

Fig. 4 shows a representative lesion.

In the lesion and control groups, body weight decreased to $85 \%$ after $11 \pm 4.1$ days and $7 \pm 1.8$ days respectively (mean \pm standard deviation) in the ABA cage.

Percentage body weight (Fig. 5A) and percentage food intake (Fig. 5B) in the lesion group are not significantly different $(\mathrm{P}=0.73, \quad \mathrm{P}=0.27$ respectively) from the control group. However, locomotor activity (Fig. 5C) is significantly lower (P $=0.037)$ in the lesion group compared to the control group. The distances run per day were $2.0 \mathrm{~km}( \pm 1.2$ $\mathrm{km})$ in the control group and $1.2 \mathrm{~km}( \pm 1.1 \mathrm{~km})$ in the lesion group (distances are means $( \pm$ standard deviations) per group, calculated from raw data averaged per rat).

Log-rank survival analysis indicates no significantly increased survival of the lesioned animals compared to controls ( $\mathrm{P}=0.598)$ (Fig. 6).

\section{DISCUSSION}

In the current study, we were able to prevent excessive running activity in the ABA model with electrolytic MD lesions. However, a curative lesion

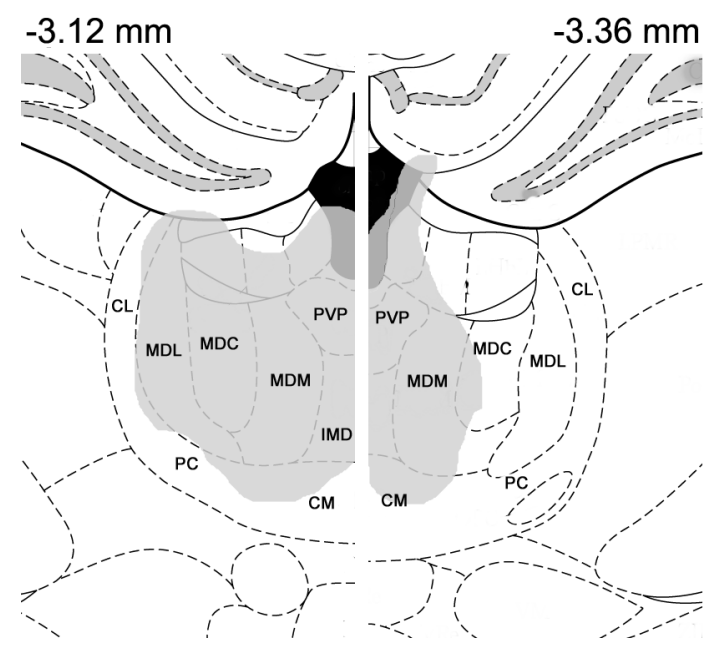

Fig. 4: Coronal brain sections depicting the electrolytic lesions (gray) on the left and right side of a rat with a representative lesion volume. The coronal section showing the largest lesion dimensions was depicted for each side. The distance of these sections posterior to Bregma is indicated. Figures modified from [27]. CL: centrolateral thalamic nucleus; $\mathrm{CM}$ : central medial thalamic nucleus; IMD: intermediodorsal thalamic nucleus; MDC: mediodorsal thalamic nucleus, central part; MDL: mediodorsal thalamic nucleus, lateral part; MDM: mediodorsal thalamic nucleus, medial part; PC: paracentral thalamic nucleus; PVP: paraventricular thalamic nucleus, posterior part.

or electrical stimulation in the MD of rats with fully developed activity anorexia, had no effect on this hyperactivity. We observed no increase in survival, body weight and food intake, neither with preventive or curative MD lesions, nor with electrical stimulation in the MD. As we were only able to modulate the hyperactivity, and not the other principal parameters in the model, the MD might not be the optimal target for therapeutic neuromodulation in anorexia nervosa. However, other core symptoms of anorexia nervosa, like the disturbance of body weight and shape perception, could not be investigated in the animal model. 



Fig. 5: Estimates of the mean ( $t$ standard error) percentage body weight (A), percentage food intake (B) and $\log$ rotations (C) in both groups.

* Significantly different from the control group at $\mathrm{P}<0.05$.

\subsection{Selection of target and stimulation parameters}

The strategy of choosing a target for stimulation based on functional neuroimaging has previously been proven successful in humans. Based upon the observation that the subgenual cingulate region was metabolically overactive in patients with treatmentresistant major depressive disorder compared to controls, six patients were electrically stimulated in the white matter tracts adjacent to the subgenual cingulate gyrus. Chronic stimulation induced a striking and sustained remission of depression in four of them [21]. According to the same line of reasoning, the posterior hypothalamus was chosen as a stimulation target in a patient with severe intractable chronic cluster headache [5]. Electrical stimulation produced complete and long-term pain relief without major side effects.

In the present study, the MD was selected because of its increased metabolism in a preceding PET study in rats in the ABA model compared to healthy controls [35]. This PET study presented valuable insights into the metabolic changes due to the activity anorexia, but the results of the present study do not convincingly support the hypothesized efficacy of lesioning or high-frequency stimulation of the hyperactive MD. One plausible explanation for the lack of effects of an electrolytic lesion or electrical stimulation may be that FDG-PET scanning is performed under general anesthetic instead of in the awake state. It is possible that FDG-PET activity measured under general anesthetic does not optimally represent brain activity related to the neurocircuitry in the model. In addition, pentobarbital anaesthesia, although administered in both ABA and control groups, might produce region-specific metabolic changes, thereby concealing the true activity in the neural circuitry of AN $[11,20]$.

Electrical stimulation was performed with a frequency of $100 \mathrm{~Hz}$. Although higher frequencies are used for motor disorders (e.g. 130 to $180 \mathrm{~Hz}$ in Parkinson's disease), stimulation with $100 \mathrm{~Hz}$ induced symptom-relief in patients suffering from treatment-resistant obsessive-compulsive disorder, a psychiatric disorder that is highly co-morbid in patients with AN [25]. Moreover, it is observed in other brain areas (e.g. ventral tegmental area and lateral hypothalamus) that electrical stimulationinduced increases in food intake level off with frequencies of 50 to $100 \mathrm{~Hz}[18,38]$. 


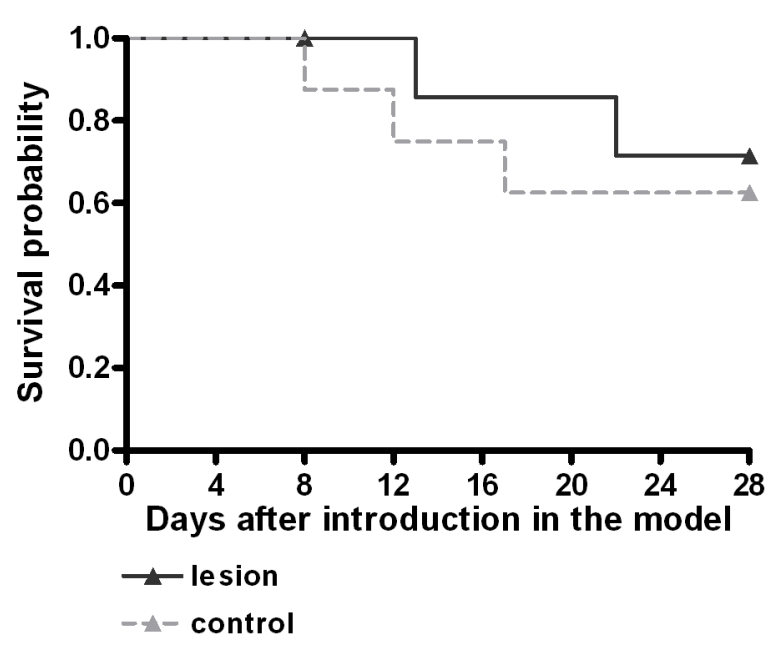

Fig. 6: Kaplan-Meier plot illustrating survival probability at daily intervals over a 28-day intervention period. In each group, eight rats were introduced in the model. Censored observations are indicated with a triangle.

\subsection{Relation MD with AN}

The MD is part of a cortico-striato-thalamocortical circuitry: In this circuitry, which has previously been linked to neuropsychiatric disorders like obsessive-compulsive disorder, the cingulate cortex projects to the ventral striatum (including the nucleus accumbens), which in turn projects via the ventral pallidum to the MD, which closes the loop by sending projections to the cingulate cortex [22]. The cingulate cortex and the ventral striatum are also involved in the ABA model [36]. Neuromodulation in brain areas of this circuitry in patients with obsessive-compulsive disorder may relieve symptoms [24]. Furthermore, a bilateral stereotactic thalamotomy induced weight gain in 2 patients suffering from extremely severe, chronic and refractory AN [40].

\subsection{Relation MD with motor activity}

An electrolytic MD lesion, applied before introduction in the ABA model, decreased locomotor activity in rats in the ABA model in experiment 2.

The MD appears to be an important component of the neural circuitry involved in regulating spontaneous and psychostimulant-induced motor activity [23]. The MD receives input from structures closely related to the basal ganglia $[7,14]$ and has robust reciprocal connections with the prelimbic prefrontal cortex, which, in turn, innervates nucleus accumbens and the ventral tegmental area [7]. Both prefrontal cortex and nucleus accumbens receive dopaminergic input, and axon terminals from MD and ventral tegmental area converge on the same pyramidal cells in deep layers of the rat prelimbic prefrontal cortex [16], suggesting that the MD is in a strong position to regulate prefrontal cortex dopamine function and motor activity.

Indeed, pharmacological blockade of glutamatergic projections from MD to prefrontal cortex has been shown to decrease prefrontal dopamine transmission, increase dopamine transmission in the nucleus accumbens and enhance locomotion [13, 14]. Also injections in the MD of a mu-opioid receptor agonist elicited a dose dependent increase in motor activity [15].

However, MD lesioning studies have yielded conflicting data on motor activity. For instance, excitotoxic MD lesions in rats increased spontaneous motor activity [10, 39]. In contrast, ibotenic MD lesions did not change the motor activity in rats in response to mild stress and amphetamine. [17]. Also electrolytic MD lesions in rats had no effect on baseline locomotion, however, they did diminish 
the locomotor activation produced by intracerebral injection of the gamma-aminobutyric acid antagonist picrotoxin into the ventral pallidum [33]. Bilateral MD lesions also reduce the locomotor response observed following stimulation of dopamine receptors in the nucleus accumbens [32].

\subsection{Outcome differences between experiment 1 and 2}

Outcome differences between experiment 1 and 2 could be attributed to different experimental designs. In experiment 2 we used male instead of female rats. Doerries found that male rats were more susceptible to the ABA model than were female rats since they reached the weight loss criterion more rapidly and ate less [3]. In addition, using male rats excluded potential effects of the strong behavioural rhythms that female rats display during the oestrus cycle [4]. Although the female rats were synchronized in experiment 1 , the fluctuating oestrus cycle still might have influenced food intake and motor activity. Also the time of intervention i.e. the start of stimulation or making the lesion (at $85 \%$ of initial body weight for experiment 1 and at surgery for experiment 2) could be a factor of variation. In experiment 2 , the lesion could already have an effect during ABA development, which was not the case in the first experiment. Due to the experimental design, the follow-up was much longer in experiment 2 (16 to 35 days) compared to experiment 1 ( 2 to 10 days).

\section{CONCLUSION}

In the current study, neither an electrolytic lesion nor high-frequency electrical stimulation affected behaviour in the activity-based anorexia model. Future research should further validate the novel approach of identifying treatment targets based on functional neuroimaging in animal models. Either other stimulation parameters could be tested in the MD or other brain areas within the circuitry revealed by functional neuroimaging could be investigated.

\section{ACKNOWLEDGEMENTS}

We acknowledge the financial support of the Research Fund K.U.Leuven (project VIS/02/007 and OT/03/57), the Institute for the Promotion of Innovation by Science and Technology in Flanders (IWT) (project SBO50151) and the Research Foundation - Flanders (FWO) (project G.0598.06). L. Luyten is a doctoral fellow of the FWO and M. Welkenhuysen is a doctoral fellow of the IWT (no. 61236).

\section{REFERENCES}

[1] American Psychiatric Association, Diagnostic and statistical manual of mental disorders : DSM-IV-TR. 4th ed. 2000, Washington, DC: American Psychiatric Association. 943 p.

[2] H.D. Burns, K. Van Laere, S. Sanabria-Bohorquez, T.G. Hamill, G. Bormans, W.S. Eng, R. Gibson, C. Ryan, B. Connolly, S. Patel, S. Krause, A. Vanko, A. Van Hecken, P. Dupont, I. De Lepeleire, P. Rothenberg, S.A. Stoch, J. Cote, W.K. Hagmann, J.P. Jewell, L.S. Lin, P. Liu, M.T. Goulet, K. Gottesdiener, J.A. Wagner, J. de Hoon, L. Mortelmans, T.M. Fong,R.J. Hargreaves, [18F]MK-9470, a positron emission tomography (PET) tracer for in vivo human PET brain imaging of the cannabinoid-1 receptor. Proc Natl Acad Sci U S A, 2007. 104(23): 9800-5. 
[3] L. Doerries, Gender differences in activity anorexia: predictable, paradoxical, or enigmatic., in Activity anorexia, theory, research, and treatment., P.W. Epling WF, Editor. 1996, Lawrence Erlbaum Associates, Inc., Publishers: Mahwah, New Jersey. 69-77.

[4] L.A. Eckel, T.A. Houpt,N. Geary, Spontaneous meal patterns in female rats with and without access to running wheels. Physiol Behav, 2000. 70(3-4): 397-405.

[5] A. Franzini, P. Ferroli, M. Leone,G. Broggi, Stimulation of the posterior hypothalamus for treatment of chronic intractable cluster headaches: first reported series. Neurosurgery, 2003. 52(5): 1095-9; discussion 1099101.

[6] C. Grillon, R. Ameli, A. Goddard, S.W. Woods,M. Davis, Baseline and fear-potentiated startle in panic disorder patients. Biological Psychiatry, 1994. 35(7): 431-439.

[7] H.J. Groenewegen,M. Trimble, The ventral striatum as an interface between the limbic and motor systems. CNS Spectr, 2007. 12(12): 887-92.

[8] E. Gutierrez, R. Vazquez,R.A. Boakes, Activity-based anorexia: ambient temperature has been a neglected factor. Psychon Bull Rev, 2002. 9(2): 239-49.

[9] J.J. Hillebrand, M.P. Koeners, C.E. de Rijke, M.J. Kas,R.A. Adan, Leptin treatment in activity-based anorexia. Biol Psychiatry, 2005. 58(2): 165-71.

[10] P.R. Hunt,J.P. Aggleton, Neurotoxic lesions of the dorsomedial thalamus impair the acquisition but not the performance of delayed matching to place by rats: a deficit in shifting response rules. J Neurosci, 1998. 18(23): 10045-52.

[11] T. Itoh, S. Wakahara, T. Nakano, K. Suzuki, K. Kobayashi,O. Inoue, Effects of anesthesia upon 18F-FDG uptake in rhesus monkey brains. Ann Nucl Med, 2005. 19(5): 373-7.

[12] J. Johansen,M. Schalling, Animals models of eating disorders, in Biological psychiatry, D'Haenen H, den Boer JA,W. P., Editors. 2002, John Wiley \& Sons, Ltd. 1117-25.

[13] M.W. Jones, I.C. Kilpatrick,O.T. Phillipson, Thalamic control of subcortical dopamine function in the rat and the effects of lesions applied to the medial prefrontal cortex. Brain Res, 1988. 475(1): 8-20.

[14] P.W. Kalivas, L. Churchill,A. Romanides, Involvement of the pallidal-thalamocortical circuit in adaptive behavior. Ann N Y Acad Sci, 1999. 877: 64-70.

[15] M.A. Klitenick,P.W. Kalivas, Behavioral and neurochemical studies of opioid effects in the pedunculopontine nucleus and mediodorsal thalamus. J Pharmacol Exp Ther, 1994. 269(1): 437-48.

[16] M. Kuroda, K. Murakami, H. Igarashi,A. Okada, The convergence of axon terminals from the mediodorsal thalamic nucleus and ventral tegmental area on pyramidal cells in layer $\mathrm{V}$ of the rat prelimbic cortex. Eur $\mathrm{J}$ Neurosci, 1996. 8(7): 1340-9.

[17] B.K. Lipska, S. Luu, N.D. Halim,D.R. Weinberger, Behavioral effects of neonatal and adult excitotoxic lesions of the mediodorsal thalamus in the adult rat. Behav Brain Res, 2003. 141(2): 105-11.

[18] M. Maliszewska-Scislo,W. Trojniar, Effect of unilateral injection of MK-801 into the area of A10 cells on feeding evoked by stimulation of homologous area in the contralateral hemisphere. Acta Neurobiol Exp (Wars), 2000. 60(4): 489-94.

[19] F.K. Marcondes, F.J. Bianchi,A.P. Tanno, Determination of the estrous cycle phases of rats: some helpful considerations. Braz J Biol, 2002. 62(4A): 609-14.

[20] A. Matsumura, S. Mizokawa, M. Tanaka, Y. Wada, S. Nozaki, F. Nakamura, S. Shiomi, H. Ochi,Y. Watanabe, Assessment of microPET performance in analyzing the rat brain under different types of anesthesia: comparison between quantitative data obtained with microPET and ex vivo autoradiography. Neuroimage, 2003. 20(4): 2040-50.

[21] H.S. Mayberg, A.M. Lozano, V. Voon, H.E. McNeely, D. Seminowicz, C. Hamani, J.M. Schwalb,S.H. Kennedy, Deep brain stimulation for treatment-resistant depression. Neuron, 2005. 45(5): 651-60.

[22] M. Mega,J. Cummings, Frontal subcortical circuits. Anatomy and function, in The frontal lobes and neuropsychaitric illness, M.P. Salloway S., Duffy J., Editor. 2001, American psychiatric publishing: Washington DC.

[23] G.J. Mogenson, S.M. Brudzynski, M. Wu, C.R. Yang,C.C.Y. Yim, Limbic motor circuits and neuropsychiatry: from motivation to action, in A review of dopaminergic regulation of limbic-nucleus accumbens-ventral pallidum-pedunculopontine nucleus circuitries involved in limbic-motor integration, P.W. Kalivas,C.D. Barnes, Editors. 1993, CRC Press: Boca raton, FL. 193-236.

[24] B. Nuttin, P. Cosyns, H. Demeulemeester, J. Gybels,B. Meyerson, Electrical stimulation in anterior limbs of internal capsules in patients with obsessive-compulsive disorder. Lancet, 1999. 354(9189): 1526.

[25] B.J. Nuttin, L.A. Gabriels, P.R. Cosyns, B.A. Meyerson, S. Andreewitch, S.G. Sunaert, A.F. Maes, P.J. Dupont, J.M. Gybels, F. Gielen,H.G. Demeulemeester, Long-term electrical capsular stimulation in patients with obsessive-compulsive disorder. Neurosurgery, 2003. 52(6): 1263-72; discussion 1272-4.

[26] K.M. O'Brien,N.K. Vincent, Psychiatric comorbidity in anorexia and bulimia nervosa: nature, prevalence, and causal relationships. Clin Psychol Rev, 2003. 23(1): 57-74.

[27] G. Paxinos, The rat nervous system. 2004, San Diego: Elsevier Academic Press. 
[28] T.S. Rieg, Validity criteria for animal models of anorexia nervosa involving activity anorexia, in Activity anorexia: theory, research and treatment, W.F. Epling,W.D. Pierce, Editors. 1996, Lawrence Erlbaum Associates: Mahwaw, New Jersey.

[29] A. Routtenberg,A.W. Kuznesof, Self-starvation of rats living in activity wheels on a restricted feeding schedule. J Comp Physiol Psychol, 1967. 64(3): 414-21.

[30] R.K. Stoving, J. Hangaard, M. Hansen-Nord,C. Hagen, A review of endocrine changes in anorexia nervosa. J Psychiatr Res, 1999. 33(2): 139-52.

[31] M. Strober, The future of treatment research in anorexia nervosa. Int J Eat Disord, 2005. 37 Suppl: S90-4.

[32] N.R. Swerdlow,G.F. Koob, The neural substrates of apomorphine-stimulated locomotor activity following denervation of the nucleus accumbens. Life Sci, 1984. 35(25): 2537-44.

[33] N.R. Swerdlow,G.F. Koob, Lesions of the dorsomedial nucleus of the thalamus, medial prefrontal cortex and pedunculopontine nucleus: effects on locomotor activity mediated by nucleus accumbens-ventral pallidal circuitry. Brain Res, 1987. 412(2): 233-43.

[34] D.L. Tempel, G. Shor-Posner, D. Dwyer,S.F. Leibowitz, Nocturnal patterns of macronutrient intake in freely feeding and food-deprived rats. Am J Physiol, 1989. 256(2 Pt 2): R541-8.

[35] K. van Kuyck, C. Casteels, P. Vermaelen, G. Bormans, B. Nuttin,K. Van Laere, Motor- and food-related metabolic cerebral changes in the activity-based rat model for anorexia nervosa: a voxel-based microPET study. Neuroimage, 2007. 35(1): 214-21.

[36] K. van Kuyck, C. Casteels, P. Vermaelen, G. Bormans, B. Nuttin,K. Van Laere, Motor- and food-related metabolic cerebral changes in the activity-based rat model for anorexia nervosa: A voxel-based microPET study. Neuroimage, 2007. 35(1): 214-221.

[37] G. Verbeke,G. Molenberghs, Linear mixed models for longitudinal data. 2000, New York: Springer-Verlag. [38] M.A. Waraczynski,J.M. Kaplan, Frequency-response characteristics provide a functional separation between stimulation-bound feeding and self-stimulation. Physiol Behav, 1990. 47(5): 843-51.

[39] R. Weissenborn, R.B. Whitelaw, T.W. Robbins,B.J. Everitt, Excitotoxic lesions of the mediodorsal thalamic nucleus attenuate intravenous cocaine self-administration. Psychopharmacology (Berl), 1998. 140(2): 225-32.

[40] R. Zamboni, V. Larach, M. Poblete, R. Mancini, H. Mancini, V. Charlin, F. Parr, C. Carvajal,R. Gallardo, Dorsomedial thalamotomy as a treatment for terminal anorexia: a report of two cases. Acta Neurochir Suppl (Wien), 1993. 58: 34-5.

[41] S. Zipfel, B. Lowe, D.L. Reas, H.C. Deter,W. Herzog, Long-term prognosis in anorexia nervosa: lessons from a 21-year follow-up study. Lancet, 2000. 355(9205): 721-2. 